\title{
Velocity Fields of External and Boundary-Layer Flows during Run-up Phase of a Solitary Wave Traveling on a Sloping Beach
}

\author{
[Chang Lin, Wei-Ying Wong, Ming-Jer Kao ]
}

\begin{abstract}
The characteristics of velocity fields of the external and boundary layer flows during run-up motion of a non-breaking solitary wave propagating over a 1:3 sloping beach are explored experimentally. Flow visualization technique (FVT) of particle trajectory photography and high-speed particle image velocimetry (HSPIV) were employed to qualitatively observe and quantitatively measure the macro-viewed external and micro-viewed boundary layer flow fields. The experiments were conducted in wave flume equipped with a wave maker being triggered by a precise servo-motor. The incident wave-height to water-depth ratios, $H_{0} / h_{0}$, is equal to 0.363 . The slope of the sloping beach model is fixed at 1:3. Profoundly distinct characteristics of flow fields around the flow demarcation curves, as observed respectively from the macro-viewed external flow and the micro-viewed boundary layer flow, are first interpreted, together with elucidation of the flow reversal exhibiting wall-jet-like feature. A promising similarity profile of the flow reversal underlying the flow demarcation curve is proposed by selecting the characteristic length and velocity scales in the boundary layer flow.
\end{abstract}

Keywords - velocity field, flow visualization, high-speed particle image velocimetry, wall jet

\section{Introduction}

For a solitary wave propagating over a sloping beach, the wave shoals with continuous deformation of free surface

\section{Chang Lin \\ Department of Civil Engineering \\ National Chung Hsing University \\ Taiwan}

\section{Wei-Ying Wong}

Department of Civil Engineering

National Chung Hsing University

Taiwan

\section{Ming-Jer Kao}

Department of Civil Engineering

National Chung Hsing University

Taiwan elevation, may break at breaking point, and starts to run-up shoreward until the maximum run-up height has been reached. The water mass triggered by gravity force begins to run-down with the retreated motion, thus moving shoreward. The water mass triggered by gravity force begins to rundown with retreated motion, thus forming a (supercritical) flow with decreasing water depth and increasing flow velocity traveling down the sloping bottom. It then moves into a (subcritical) flow zone with increasing water depth and decreasing flow velocity. The investigation on a complete evolution of solitary waves thus receive utmost importance that can profoundly enhance understanding of the run-up and run-down motions as well as the shoreward inundation of a long-wave or tsunami, aiming to predict the inundation area of coastal zone for the need of disaster mitigation (Hsiao et al. [1]; Hwung et al. [2]).

Recently, employing a high speed particle image velocimetry (HSPIV), Lin et al. [3,4] investigated the velocity characteristics in the external flow as well as the near-bottom and boundary layer flow mainly in the prebreaking zone of shoaling solitary waves propagating over a 1:10 slope. New findings of both flow separation stemming from 1:10 and 1:5 sloping bottoms and subsequent vortex structures taking place underlying the separated shear layer during run-down motion, were briefly identified in Lin et al. [4,5], using FVT and HSPIV. In addition, as indicated significantly by Grilli et al. [6], variation of the beach slope will play more important role than the wave-height to waterdepth ratio of the incident solitary wave in dominating the evolutions of free surface elevation and wave height in the pre-breaking, surf and swash zones.

This study aims to perform a profound elucidation of differences in the velocity fields of external and boundarylayer flows, together with existence of flow demarcation curve, during run-up motion of a solitary wave propagating over a 1:3 steeper beach, using FVT and HSPIV.

\section{Experimental Set-ups}

The experiments were conducted in a glass-walled and glass-bottomed wave flume with dimensions of $14.0 \mathrm{~m}$ long, $0.25 \mathrm{~m}$ wide and $0.5 \mathrm{~m}$ deep. The wave flume is equipped with a piston-type wave maker, which is triggered by a precision servo-motor. Two capacitance type wave gauges 
were employed to measure the water surface elevations. The incident wave-height to water-depth ratios, $H_{0} / h_{0}$, is set at 0.363 . The sloping beach model having a slope of $1: 3$ was made of acrylic. The toe of the sloping beach was installed at the position $9.0 \mathrm{~m}$ away from the wave maker at rest.

A flow visualization technique with neutrally suspending particles was used to qualitatively observe the flow characteristics. A laser light sheet was utilized to brighten the two-dimensional motion of the suspending particles on a vertical plane. A high-speed camera was used to capture images with maximum framing rate of $1200 \mathrm{~Hz}$ and maximum image resolution of $1,024 \times 1,024$ pixel. In addition, distinct velocity fields and profiles in the external and bottom boundary-layer flows were measured by using HSPIV. During HSPIV measurements, two different sizes of field of view (FOV) for capturing the flow images were employed. The dimension of larger FOV is $17.0 \mathrm{~cm} \times 10.63$ $\mathrm{cm}$ and the counterpart of smaller FOV is $2.6 \mathrm{~cm} \times 1.3 \mathrm{~cm}$. The final global velocity field is a mosaic of some or all of the FOVs measured at different positions.

The origin $(x, y)=(0,0) \mathrm{cm}$ of the coordinate system is located at the toe of the sloping bottom with positive $x$ being in the horizontal direction of wave motion and the positive $y$ in the vertical upward direction, measured from the horizontal bottom. Herein, $t$ denotes time with $t=0$ being characterized as the instant when the crest of the solitary wave is exactly above the toe of sloping beach (i.e., the toe section) at $x=0 \mathrm{~cm}$.

\section{Results and Discussion}

The temporal and spatial variations of the ensembleaveraged velocity fields of the external flow (i.e., without taking boundary layer flow into account) during run-up motion of the solitary wave for $12.5 \mathrm{~cm} \leq x \leq 24.5 \mathrm{~cm}$ are shown in Figs. 1(a-d) for $t=0.190 \mathrm{~s}, 0.410 \mathrm{~s}, 0.475 \mathrm{~s}$ and $0.540 \mathrm{~s}$, respectively. As a reference, $t=0 \mathrm{~s}$ is defined as the corresponding time for the wave crest exactly passing through the toe section at $x=0 \mathrm{~cm}$.

The velocity field for shoaling phase of the solitary wave at $t=0.190 \mathrm{~s}$ is depicted in Fig. 1(a), showing that the wave crest appears at the section of $x=x_{\mathrm{wc}}=18.25 \mathrm{~cm}$. As seen from the velocity field, all of the water particles do move onshore with the maximum uniform velocity (in the horizontal direction), $u_{\mathrm{u}}$, being $46.4 \mathrm{~cm} / \mathrm{s}$ at $x=24.0 \mathrm{~cm}$, together with the uniform velocity, $u_{\mathrm{u}}$, being $30.2 \mathrm{~cm} / \mathrm{s}$ at $x=$ $14.0 \mathrm{~cm}$, and $37.6 \mathrm{~cm} / \mathrm{s}$ at $x=19.1 \mathrm{~cm}$. These evidence clearly shows increasing trend of the onshore uniform velocity for $t=0.136 \mathrm{~s}$, strongly highlighting existence of the convective acceleration under the favorable pressure gradient in the onshore direction. Based on the images continuously recorded during flow visualization test, run-up motion of the solitary wave is rendered to start at $t=0.271 \mathrm{~s}$ and end right at $t=0.655 \mathrm{~s}$ accompanied immediately by the commencement of run-down motion. Note that these wave-
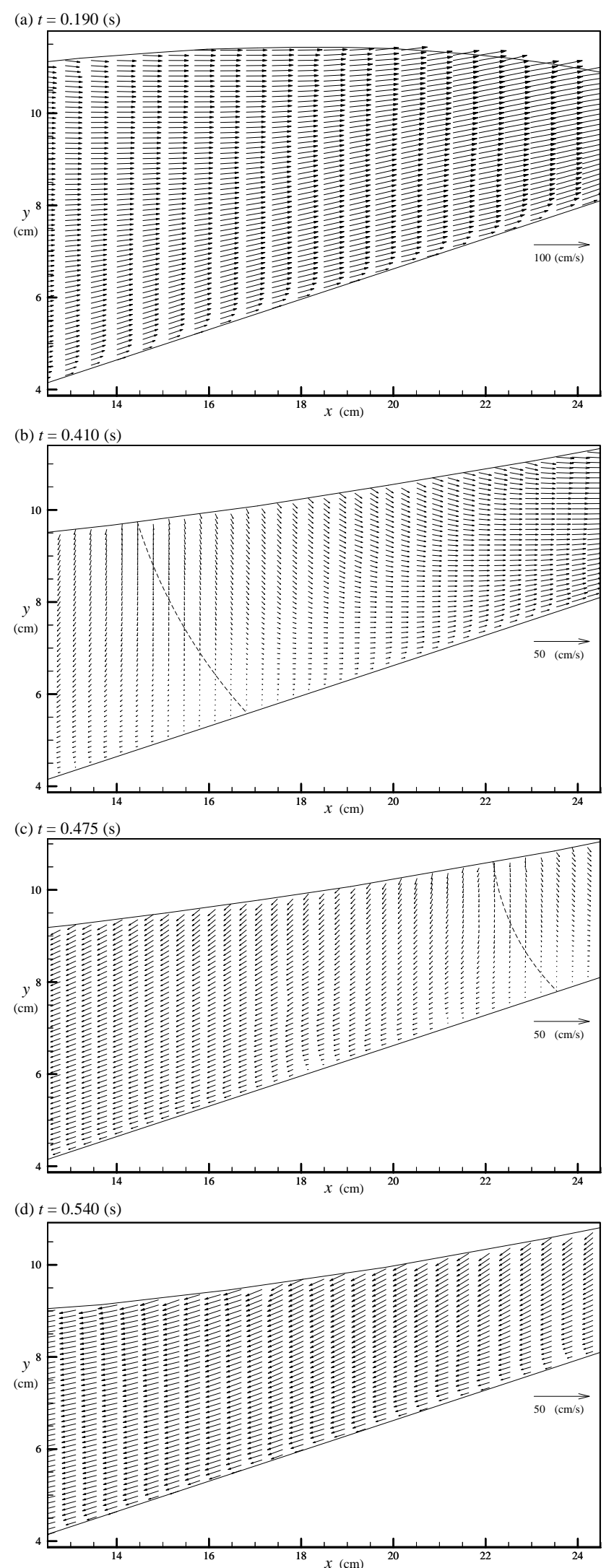

Figure 1. Distinct ensemble-averaged velocity fields obtained at four different times (a) $t=0.190 \mathrm{~s}$; (b) $t=0.410 \mathrm{~s}$; (c) $t=0.475 \mathrm{~s}$; (d) $t=0.540 \mathrm{~s}$ 
crest sections for $t \geq 0.271 \mathrm{~s}$ are located at $x_{\mathrm{wc}} \geq 26.0 \mathrm{~cm}$, which are on the onshore side of the equilibrium shoreline positioned at $x=24.0 \mathrm{~cm}$.

Figs. 1(b, c) illustrate the velocity fields at $t=0.410 \mathrm{~s}$ and $0.475 \mathrm{~s}$, revealing that the flow field occurs approximately in the middle stage of run-up phase. A novel feature in the velocity fields for $t=0.410 \mathrm{~s}$ and $0.475 \mathrm{~s}$ can be characterized as appearance of the flow bifurcation into two streams, having one moving onshore and the other runs offshore with the flow demarcation curve (marked with dashed line) in between. The flow demarcation curve emanates from the free surface toward the sloping bottom. The horizontal velocities have the positive and negative values on the onshore and offshore sides of this curve, respectively, but are all equal to zero on the curve. For $t=$ $0.410 \mathrm{~s}$ and $0.475 \mathrm{~s}$, the onshore extents of flow demarcation curve cover the intervals of $14.40 \mathrm{~cm} \leq x \leq 16.80 \mathrm{~cm}$ and $22.15 \mathrm{~cm} \leq x \leq 23.55 \mathrm{~cm}$ with the centroids of the two flow demarcation curves being positioned at $\left(x_{\mathrm{c}}, y_{\mathrm{c}}\right)=(15.40$, $7.55) \mathrm{cm}$ and $\left(x_{\mathrm{c}}, y_{\mathrm{c}}\right)=(22.60,9.10) \mathrm{cm}$, respectively.

Fig. 1(d) presents the velocity field at later run-up phase for $t=0.540 \mathrm{~s}$, demonstrating all of the water particles moving in the offshore direction. The maximum "offshore" uniform velocity, $u_{\mathrm{u}}$, has a value of $-13.7 \mathrm{~cm} / \mathrm{s}$ taking place at $x=15.0 \mathrm{~cm}$, along with the offshore uniform velocity being $-9.0 \mathrm{~cm} / \mathrm{s}$ at $x=24.0 \mathrm{~cm},-12.3 \mathrm{~cm} / \mathrm{s}$ at $x=19.1 \mathrm{~cm}$ and $-13.8 \mathrm{~cm} / \mathrm{s}$ at $x=14.0 \mathrm{~cm}$. These witness demonstrates existence of the convection deceleration in the onshore direction, i.e., the convection acceleration in the offshore direction for $t=0.540 \mathrm{~s}$. It should be mentioned that, for $t=$ $0.540 \mathrm{~s}$, the extension of flow demarcation curve in the horizontal direction has moved to the interval ranging from $x=30.40 \mathrm{~cm}$ to $x=31.90 \mathrm{~cm}$, together with both the centroid of flow demarcation curve translating to the position at $\left(x_{\mathrm{c}}, y_{\mathrm{c}}\right)=(30.90,11.10) \mathrm{cm}$.

Based on a series of velocity fields taking place at different times, the temporal variation of the ensembleaveraged horizontal velocities measured at $x=15.0 \mathrm{~cm}$ (i.e., situated on the offshore side of the equilibrium shoreline at $x$ $=24.0 \mathrm{~cm})$ is extracted and shown in Fig. 2 with $-0.160 \mathrm{~s} \leq t$ $<0.271 \mathrm{~s}$ for the pre-shoaling and shoaling phases and 0.271 $\mathrm{s} \leq t \leq 0.655 \mathrm{~s}$ for the entire run-up process. Note that the instantaneous free surface elevations (marked by the dashed line with cursors indicating the temporal variation process) are clearly plotted above all of the velocity profiles.

As shown in Fig. 2, the onshore horizontal velocities are evidenced to increase significantly with increasing $t$, say from $t=-0.160 \mathrm{~s}$ to $t=0.090 \mathrm{~s}$. In addition, with considerable rising of the free surface elevation for $0.09 \mathrm{~s} \leq t$ $<0.181 \mathrm{~s}$, the onshore horizontal velocities remain slight increase for $y^{*}=(y-x / 3)>3.73 \mathrm{~cm}$ and keep minor decrease for $y^{*}<3.62 \mathrm{~cm}$. After occurrence of the maximum free surface elevation at $t=0.181 \mathrm{~s}$, the onshore horizontal velocities, $u$, then keep decreasing for $0.181 \mathrm{~s}<t<0.410 \mathrm{~s}$ and the corresponding velocity profiles exhibit fairly linear

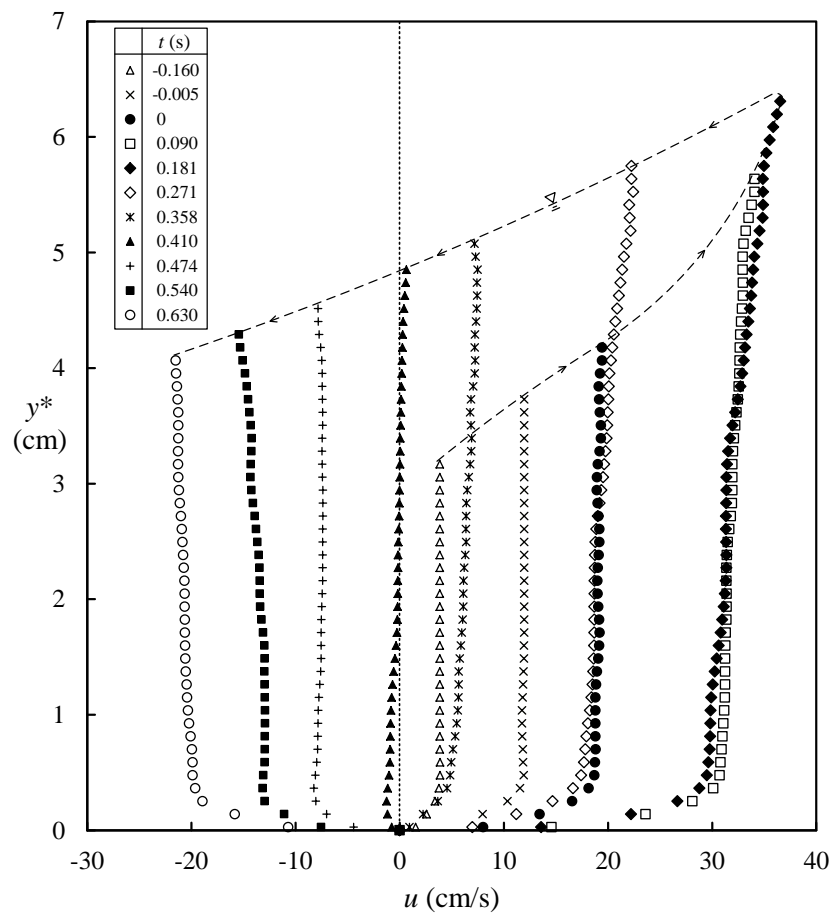

Figure 2. Ensemble-averaged horizontal velocity profiles measured at $x=$ $15.0 \mathrm{~cm}$ for eleven different times

shape, except those near the sloping bottom approximately for $y^{*}<0.4 \mathrm{~cm}$. Interestingly note that, due to existence of the flow demarcation curve crossing the measurement section at $x=15.0 \mathrm{~cm}$ for $t=0.410 \mathrm{~s}$ [see Fig. 1(b)], the upper and lower parts of the horizontal velocity profile exhibit the onshore (positive) and offshore (negative) values, respectively, with zero velocity occurring in between and being located around $y^{*}=2.94 \mathrm{~cm}$.

As time $t>0.410 \mathrm{~s}$ approximately, all of the horizontal velocities in each profile (except zero velocity right on the surface of the sloping beach) show the "offshore" (negative) values with its magnitudes increasing with $t$ even during run-up phase of the solitary wave propagating "onshore." Worthy of mentioning is that the temporal free surface elevation keeps decreasing (as evidenced along the dashed line being plotted above each velocity profile in Fig. 2) after the maximum free surface elevation have been reached at $t=$ $0.181 \mathrm{~s}$. In summary, the temporal variation of the horizontal velocity obtained at $x=15.0 \mathrm{~cm}$, in fact, experiences the local acceleration and deceleration in the "onshore" direction for $-0.160 \mathrm{~s} \leq t<0.181 \mathrm{~s}$ and $0.181 \mathrm{~s} \leq t \leq 0.630 \mathrm{~s}$, respectively. On the contrary, the induced horizontal velocity crossing the section at $x=15.0 \mathrm{~cm}$ can be interpreted as suffering the local deceleration and acceleration in the offshore direction for $-0.160 \mathrm{~s} \leq t<0.181 \mathrm{~s}$ and $0.181 \mathrm{~s} \leq t \leq$ $0.630 \mathrm{~s}$, respectively. 
To explore the velocity field near the sloping bottom, the smaller FOV for HSPIV measurements is used. The detailed velocity fields near and within the sloping-bottom boundary layer flow, which are centrally corresponding to the temporal change of the counterpart of external flow shown in Fig. 1(b), are presented in Figs. 3(a-c) for $15.0 \mathrm{~cm} \leq x \leq$ $17.5 \mathrm{~cm}$ at $t=0.403 \mathrm{~s}, 0.410 \mathrm{~s}$ and $0.417 \mathrm{~s}$, respectively.
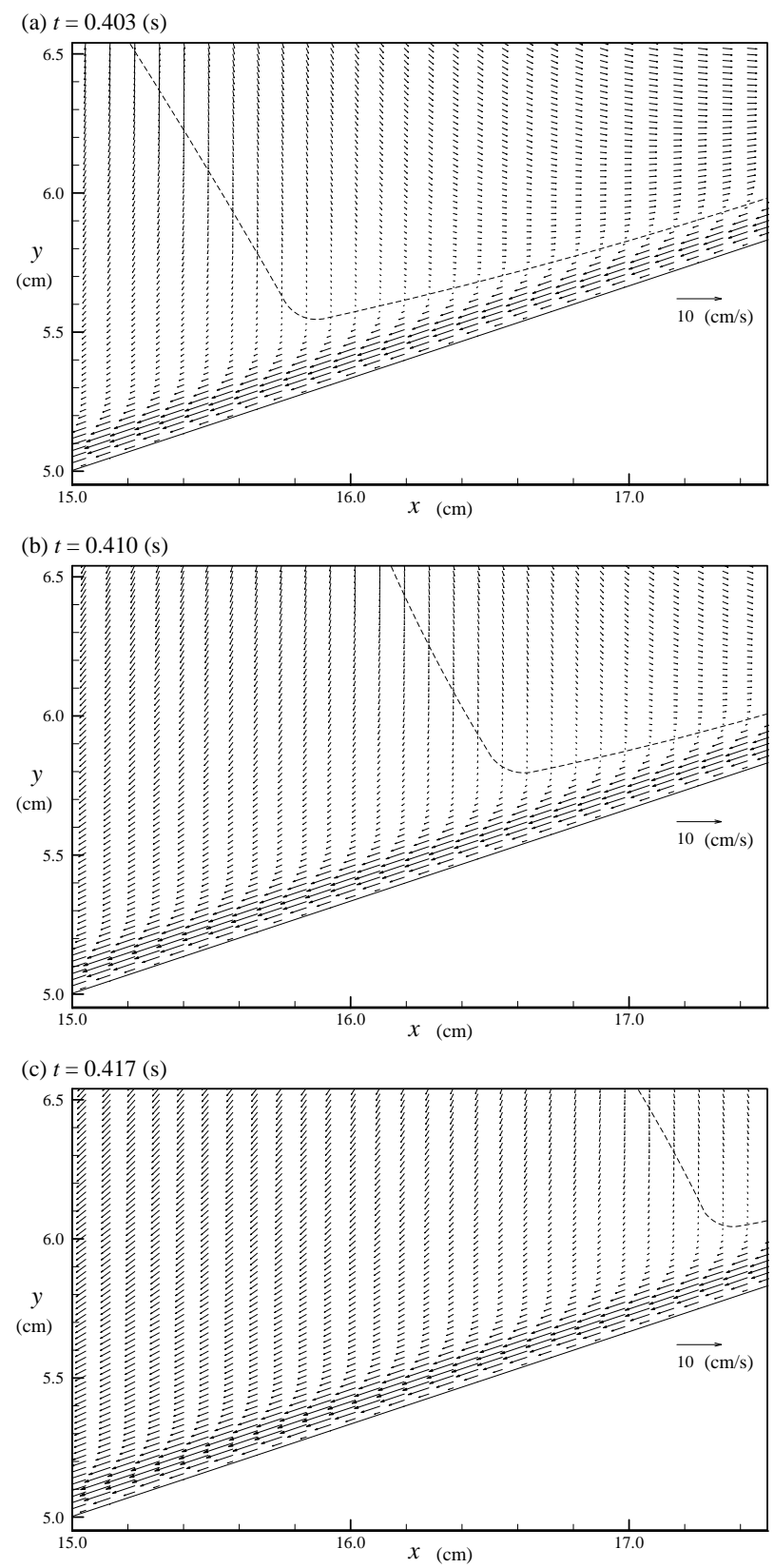

Figure 3. Ensemble-averaged velocity fields close to and in bottom boundary layer

From the view point of macro-viewed external flow and due to the non-slip condition imposed on the sloping beach, there should have a stagnation point (Salevik et al. [7]) situated on the lower end of flow demarcation curve and close to the sloping-bottom surface. Right at the stagnation point the horizontal and vertical velocities are both zero, and around this point the approaching flow bifurcates into two opposite (i.e., offshore and onshore) streams. However, from the micro-viewed flow field as depicted in Figs. 3(a-c), the stagnation point does not exist in the flow, but the flow demarcation curve takes a concave bend near the sloping bottom and then extends further onshore. The offshore stream not only stems from the water particles having offshore horizontal velocities which are located on the offshore side the flow demarcation curve [as seen in Fig. 1(b)]; but also originates from the counterparts underneath the flow demarcation curve with strong flow reversal revealing the wall-jet-like profile (Lin et al. $[4,5,8,9])$. The thickness and maximum offshore velocity of flow reversal underneath the flow demarcation curve both keep increasing in the offshore direction. Such a spectacular feature still holds true for all sections passing through the tip of the concave bend and further toward offshore position. The evidence further supporting this particular feature can be witnessed by using one of the images taken during flow visualization tests, as shown in Fig. 4.

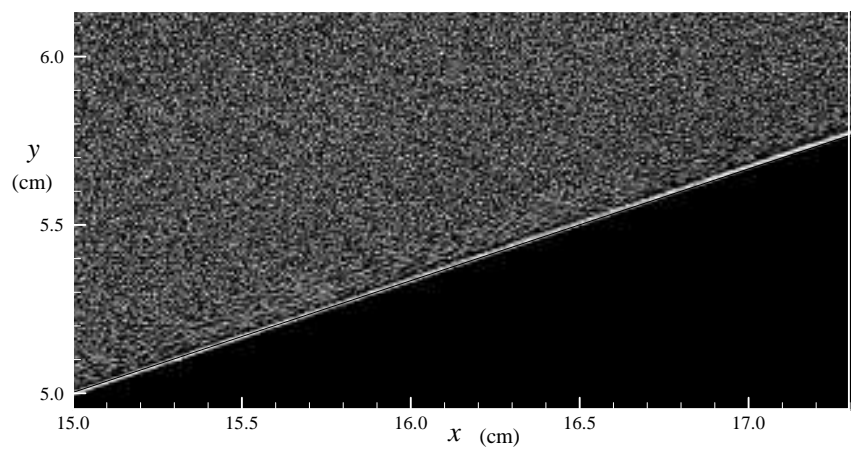

Figure 4. Flow visualized image revealing strong wall-jet-like flow right above the surface of sloping beach

Fig. 5 summarizes the temporal variation of the flow demarcation curve for $t=0.403 \sim 0.417 \mathrm{~s}$, highlighting the similar propagating feature for the three specified curves. If the centroid of global flow demarcation curve [see dotted points as illustrated in Figs. 1(b, c)] or the tip of each concave bend on a flow demarcation curve [see Fig. 5] is regarded as the representative target, then the propagation speed of the flow demarcation curve, $C_{\mathrm{fdc}}$, can be calculated.

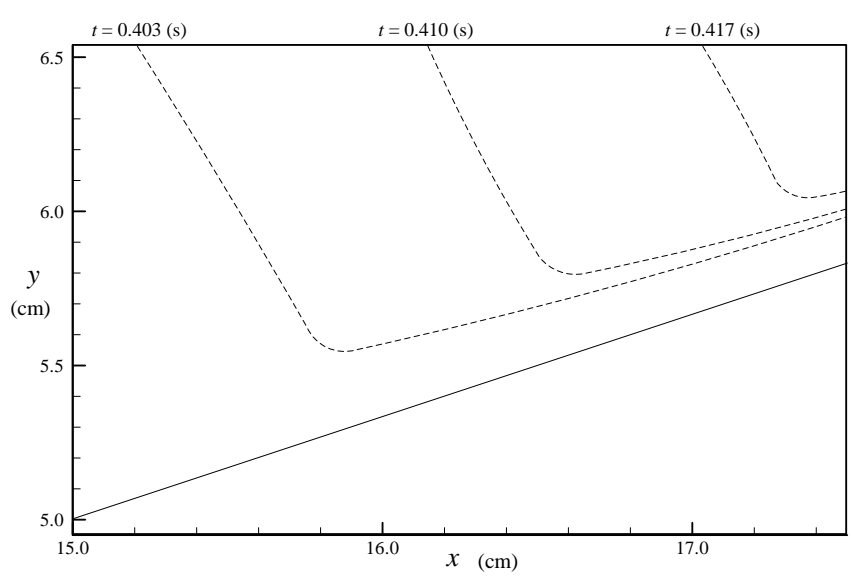

Figure 5. Temporal variation of flow demarcation curve for $t=0.403 \sim$ $0.417 \mathrm{~s}$ 
It is interestingly found that the value of $C_{\mathrm{fdc}}$ is equal to $117.9 \mathrm{~cm} / \mathrm{s}$ for $t=0.410 \mathrm{~s}$, which is higher than the counterpart of the celerity, $C_{0}$, of the incident solitary wave traveling over constant water depth region located for $x<0$ cm (i.e., $\left.C_{0}=\left[\mathrm{g}\left(H_{0}+h_{0}\right)\right]^{1 / 2}=103.4 \mathrm{~cm} / \mathrm{s}\right)$. Namely, the ratio of propagation speed of the flow demarcation curve to the wave celerity, $C_{\mathrm{fdc}} / C_{0}$, is 1.14 , which is larger than unity for $t=0.410 \mathrm{~s}$. Also note that the particular feature still holds true qualitatively for $0.410 \mathrm{~s}<t<0.655 \mathrm{~s}$.

Figs. 6(a-c) present close-ups of the horizontal velocity profiles obtained at three different positions of the tip located on the concave bend of flow demarcation curve for $t$ $=0.403 \mathrm{~s}, 0.410 \mathrm{~s}$ and $0.417 \mathrm{~s}$. Each velocity profile of the flow reversal exhibits the wall-jet-like characteristics having zero velocity right on the beach surface and the offshore maximum velocity occurring a little above the slopingbottom surface, and again zero velocity appearing at the specified height, $y_{0}$.
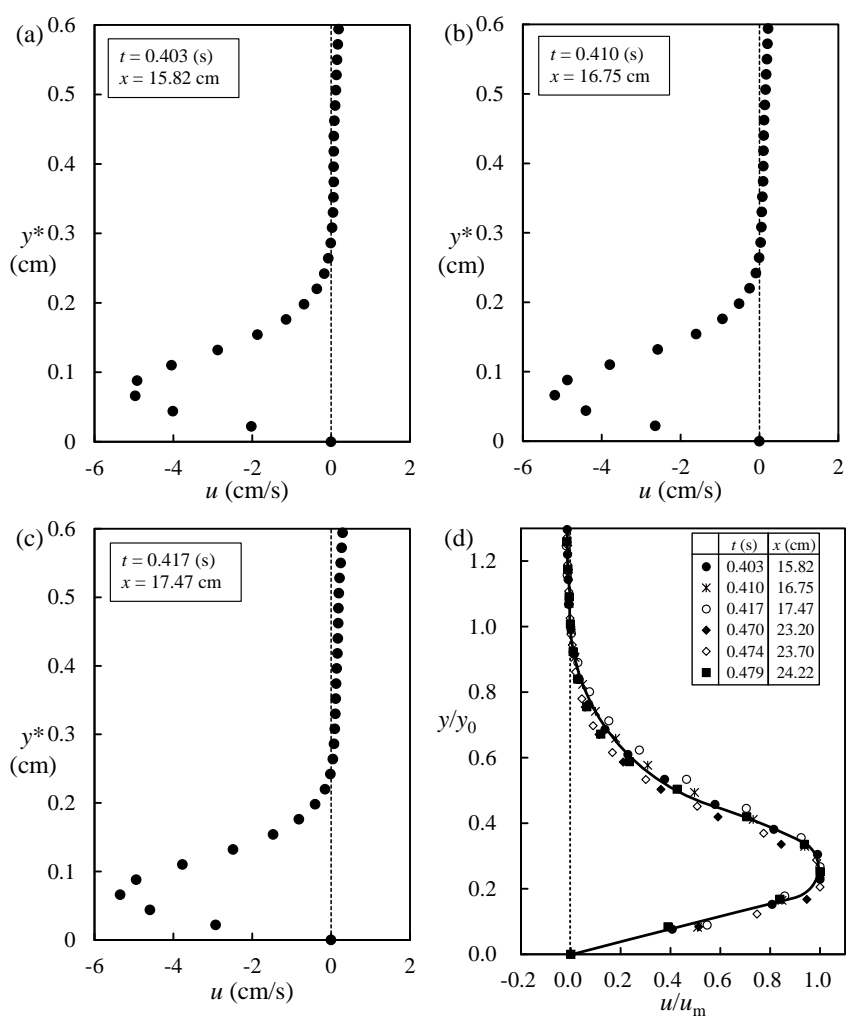

Figure 6. Close-ups of horizontal velocity profiles in boundary layer (a-c) obtained at four different positions of tip on concave bend of flow demarcation curve; (d) a similarity profile

Note that, for the velocity profile obtained at the tip of the concave bend of the flow demarcation curve, the horizontal velocities will all take the (onshore) positive values for positions located above the curves, i.e., $u>0$ for $y$ $>y_{0}$. It is surprisingly found that all of these profiles are very similar in their shapes and velocity magnitudes. By selecting the characteristic length and velocity scales using the thickness of flow reversal, $y_{0}$, and the maximum offshore horizontal velocity, $u_{\mathrm{m}}$, respectively, a non-dimensional form of $u / u_{\mathrm{m}}$ versus $y / y_{0}$ can be obtained and shown in Fig. 6(d), demonstrating existence of a promising similarity profile for the flow reversal in the boundary layer over the sloping beach.

\section{Concluding Remarks}

In the present study, the velocity fields of external and boundary layer flows during run-up phase of a solitary wave traveling over a 1:3 sloping beach have been demonstrated, using FVT and HSPIV. Prominent difference in the velocity fields around the lower end of flow demarcation curve as observed from both macro-view external flow and microview boundary layer flow has been elucidated. A promising similarity profile has been proposed for the flow reversal underneath the flow demarcation curve and right over the sloping beach, highlighting the wall-jet-like behavior in the boundary layer flow.

\section{Acknowledgment}

The authors are appreciated to the financial support from the Ministry of Science and Technology, Taiwan (No: MOST 105-2221-E-005-033-MY3) to National Chung Hsing University, Taichung, Taiwan.

\section{References}

[1] S. C. Hsiao, T. W., Hsu, T. C. Lin, and Y. H. Chang, "On the evolution and run-up of breaking solitary waves on a mild sloping beach," Coastal Engineering, vol. 55 (12), pp. 975-988, 2008.

[2] H. H. Hwung, Y. T. Wu, and C. Lin, "Tsunami propagation and related new approach of mitigation," Proceedings of the $8^{\text {th }}$ TaiwanJapan Joint Seminar on Natural Hazard Mitigation in 2015, December 7, Kyoto, Japan, 2015.

[3] C. Lin, P. H. Yeh, S. C. Hseih, Y. N. Shih, L. F. Lo, and C. P. Tsai, "Pre-breaking internal velocity field induced by a solitary wave propagating over a 1:10 slope," Ocean Engineering, vol. 80, pp. 1-12, 2014.

[4] C. Lin, P. H. Yeh, M. J. Kao, M. H. Yu, S. C. Hseih, S. C. Chang, T. R. Wu, and C. P. Tsai. "Velocity fields inside near-bottom and boundary layer flow in prebreaking zone of solitary wave propagating over a 1:10 slope," Journal of Waterways, Port, Coastal, and Ocean Engineering, ASCE, DOI: 10.106/(ASCE)WW.1943-5460.0000269 and vol. 141(3), pp. 04014038-1 30, 2015.

[5] C. Lin, M. J. Kao, G. W. Tzeng, W. Y. Wong, J. Yang, R. V. Raikar, T. R. Wu, and P. L. F. Liu, "Study on flow field of boundary-layer separation and hydraulic jump during run-down motion of shoaling solitary wave," Journal of Earthquake and Tsunami, vol. 9(5), pp. 1540002-1 33, 2015.

[6] S. T. Grilli, I. A. Svendsen, and R. Subramanya, "Breaking criterion and characterisitics for solitary waves on slopes," Journal of Waterway, Port, Coastal, and Ocean Engineering, ASCE, vol. 123(3), pp. 102-112, 1997.

[7] G. Salevik, A. Jensen, and G. Pedersen, "Runup of solitary waves on a straight and a composite beach." Coastal Engineering, vol. 77, pp. 40-48, 2013.

[8] C. Lin, S. C. Hsieh, Y. R. Lin, K. A. Chang, and R. V. Raikar, "Flow property and self-similarity in steady hydraulic jumps," Experiments in Fluids, vol. 53, pp. 1591-1616, 2012.

[9] C. Lin, S. M. Yu, W. Y. Wong, G. W. Tzeng, M. J. Kao, P. H. Yeh, R. V. Raikar, J. Yang, and C. P. Tsai, "Velocity characteristics in boundary layer flow caused by solitary wave traveling over horizontal bottom," Experimental Thermal and Fluid Science, vol. 76, pp. 238252, 2016. 